\title{
Diastole and its Beneficial Role in Coronary Atherogenesis
}

\author{
*JV Soulis, GD Giannoglou, TM Farmakis, GE Louridas \\ *Fluid Mechanics Division, Democrition University of Thrace, Xanthi, Greece, \\ AHEPA University Hospital, Aristotle University of Thessaloniki, Greece
}

\begin{abstract}
The purpose of this study is to elucidate the role, which Wall Shear Stress (WSS) differentiation between diastole and systole plays in coronary atherosclerosis. A finite-element analysis of the $3 D$, pulsatile, nonNewtonian, heamodynamics of the normal human left main coronary artery bifurcation, based on published data, is performed. The time averaged mean WSS in the entire left coronary artery bifurcation region ranged from 0.086 to $5.97 \mathrm{~N} / \mathrm{m} 2$. Arterial WSS was significantly lower on the lateral walls of the bifurcation, for all tested time steps of the pulse cycle. The distribution of low WSS along the walls is in agreement with the common locations of atheroma. It is the systolic period, rather than the diastolic one, which is probably associated with the development of atherosclerotic plaques, due to significantly lower WSS values during systole.
\end{abstract}

\section{Introduction}

Blood flow in arterial bifurcation segments is of paramount importance in understanding the biomedical pathophysiology of atherosclerosis. Via the application of the CFD, as it is applied to human coronary artery data, is possible to quantitatively analyze the local haemodynamic parameters, particularly the wall shear stress, in order to elucidate the blood flow patterns in regions where atherosclerosis usually appears (bifurcations and trifurcations). Particularities in human coronary geometry also play an important role in the atherosclerosis development. Flow separation, recirculation as well as low and oscillating wall shear stress are decisive atheromatic factors. Secondary flows near the arterial wall are also expected to occur along curved vessels as a result of acting centrifugal forces.

Numerical studies of pulsatile flows through coronary bifurcations with emphasis on wall shear stress effects were carried out by Tu et al. [1] who analyzed flows with stenosis and $\mathrm{He}$ and $\mathrm{Ku}$ [2] who treated the blood as Newtonian fluid and performed analysis in the left coronary artery bifurcation. They concluded that the highly localized distribution of low and oscillatory shear stress along the walls strongly correlates with focal locations of atheroma in this type of artery. The importance of non-Newtonian viscosity distribution in the human coronary artery was reported by Gijsen et al [3] and Giannoglou et al. [4].

The aim of the present study was to numerically analyze the three-dimensional, non-Newtonian, pulsatile flow in normal human left main coronary artery bifurcation model, applying a finite-element technique in order to further investigate the spatial and time wall shear stress distribution. Emphasis was put into wall shear distribution during systole and diastole, in order to elucidate the role, which WSS differentiation between diastole and systole plays in coronary atherosclerosis. The results shed light into the three-dimensional nature of the flow patterns and quantify the distribution of wall shear stress particularly in regions close to the flow divider and opposite to it areas.

\section{Methods}

The geometry used for the construction of the left main coronary artery model is reported by $\mathrm{He}$ and $\mathrm{Ku}$ [2] and was based on data compiled from several sources. The human left coronary artery is divided into three parts: the left main coronary artery (LMCA), and the two branches namely the left anterior descending branch (LAD) and the left circumflex branch (LCX). The average dimensions in lengths and diameters for the left coronary bifurcation artery model are as follows: length of LMCA is $11.0 \mathrm{~mm}$, while the cross-section diameters are 4.0, 3.4 and $3.0 \mathrm{~mm}$ for the LMCA, LAD and LCX, respectively. The angle between $\mathrm{LMCA}$ and $\mathrm{LAD}$ is $159^{\circ}$, between LMCA and LCX $117^{\circ}$ and finally between LAD and LCX $84^{\circ}$. The LMCA is a straight tube. The LAD and LCX are curved tubes with constant radii of curvature with $42.8 \mathrm{~mm}$ and $39.3 \mathrm{~mm}$, respectively. The bifurcation plane is located in one plane namely the bifurcation plane, while the curvature planes for the LAD and LCX are perpendicular to the bifurcation plane.

The geometry data were input to a pre-processing code for unstructured mesh generation. A total of 61,635 tetrahedral cells corresponding to 14,147 nodes were used for the final CFD analysis. The above number of cell was considered to be satisfactory for WSS analysis. Figure 1 shows the unstructured mesh of the tested human left coronary artery. 
All mesh and flow data were transferred into the main CFD solver FLUENT (3D double precision version, release 5.5.14, Fluent Inc., Lebanon, NH). The numerical code solves the governing Navier-Stokes flow equations. The assumptions made about the flow are threedimensional, unsteady and laminar, while the arterial vessel walls are inelastic and impermeable. The blood is treated as non-Newtonian fluid obeying to the power law.

In order to simulate the physiological phasic flow condition, a typical normal phasic waveform of the left coronary artery system (referring to resting conditions), shown in Figure 2, is applied over the entire entrance cross-section. A total of 200 time-steps were applied to elucidate the flow behaviour throughout the cardiac cycle. At each time-step, the velocity is considered to be uniform over the entrance cross-section (plug inlet flow profile). The most characteristic points of cardiac cycle were selected for further analysis. Six points of the left coronary artery phasic blood flow were selected for detailed presentation and analysis corresponding to the most characteristic points of the cardiac cycle.

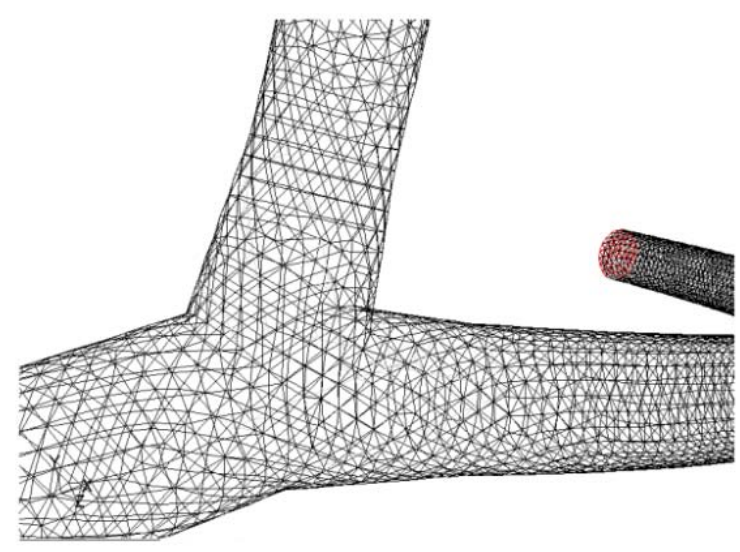

Figure 1. Unstructured mesh of the tested human left coronary artery. Mesh details near to bifurcation region.

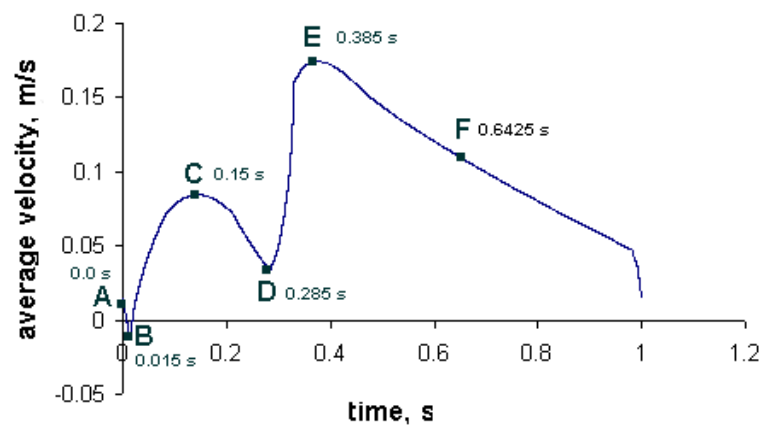

Figure 2. Phasic left coronary artery velocity

\section{Results}

Emphasis was put into the wall shear distribution during systole and diastole. Two definitions for the cardiac cycle exist: for cardiologists and for physiologists. The most characteristic points A, B, C, D, $\mathrm{E}$, and $\mathrm{F}$ of either phase definitions is shown in Figure 2. In detail, point $\mathrm{A}$ refers to the start of the physiological cardiac cycle, representing the start of isovolumic contraction where left ventricular pressure crosses over atrial pressure. Point B refers to the first heart sound (mitral closure), which is the start of the cardiac cycle for the cardiologist, occurs at about $15 \mathrm{msec}$ after the onset of physiological systole. Point $\mathrm{C}$ occurs $150 \mathrm{msec}$ after the crossover of pressure referring to the peak of systole. This is also the end of physiological systole (peak of the ejection phase). Point D occurs $285 \mathrm{msec}$ after the onset of systole, simultaneously with the insicura of the aortic pressure waveform (closure of the aortic valve), and this is the end of cardiological systole. Point E occurs $385 \mathrm{msec}$ after point $\mathrm{A}$, at the peak of diastolic left coronary blood flow. Finally, point F, occurring $642 \mathrm{msec}$ after the onset of physiological cardiac cycle, represents the middle of the cardiological diastole phase.

Wall shear stress in the entire bifurcation region ranged from 0.086 to $5.97 \mathrm{~N} / \mathrm{m}^{2}$ for all tested times of the cardiac cycle. At the flow divider (inner wall of the bifurcation), maximum wall shear stress was $2.69 \mathrm{~N} / \mathrm{m}^{2}$ at systole and $5.97 \mathrm{~N} / \mathrm{m}^{2}$ at diastole and remained unidirectional during systole. At the outer wall of the left coronary artery bifurcation the instantaneous wall shear stress oscillated between 0.086 and $5.82 \mathrm{~N} / \mathrm{m}^{2}$. For all tested time steps of the pulsatile waveform the flow divider region encounters higher wall shear stress distribution than the lateral walls.

At point $\mathrm{A}$, the minimum wall shear stress values in the entire bifurcation region were of the order of $0.086 \mathrm{~N} / \mathrm{m}^{2}$. The maximum wall shear stress was $2.68 \mathrm{~N} / \mathrm{m}^{2}$ while the average value in this region was $1.13 \pm 0.021 \mathrm{~N} / \mathrm{m}^{2}$.

At point $\mathrm{B}$, minimum computed negative WSS values in the entire region were of the order of $0.35 \mathrm{~N} / \mathrm{m}^{2}$. Note, that the pulsatile waveform attains negative velocity throughout. The maximum negative WSS was $0.74 \mathrm{~N} / \mathrm{m}^{2}$ while the average value in this region was $0.50 \pm 0.025 \mathrm{~N} / \mathrm{m}^{2}$.

Thereafter, the entrance velocity increases and at $150 \mathrm{msec}$, point $\mathrm{C}$, i.e. at the start of the cardiac cycle, the first maximum velocity value of $0.085 \mathrm{~m} / \mathrm{s}$ was recorded. In this case, the minimum WSS value in this region was of the order $0.92 \mathrm{~N} / \mathrm{m}^{2}$ while the maximum WSS was $2.75 \mathrm{~N} / \mathrm{m}^{2}$ and the average value was $1.75 \pm 0.015 \mathrm{~N} / \mathrm{m}^{2}$. The flow now is unidirectional and all wall shear stress values were positive. The lateral walls exhibit lower minimum WSS values than the flow divider region. Thus, the minimum value of encountered on the lateral wall 
was $0.92 \mathrm{~N} / \mathrm{m}^{2}$ while the minimum value on the flow divider region was $1.45 \mathrm{~N} / \mathrm{m}^{2}$.

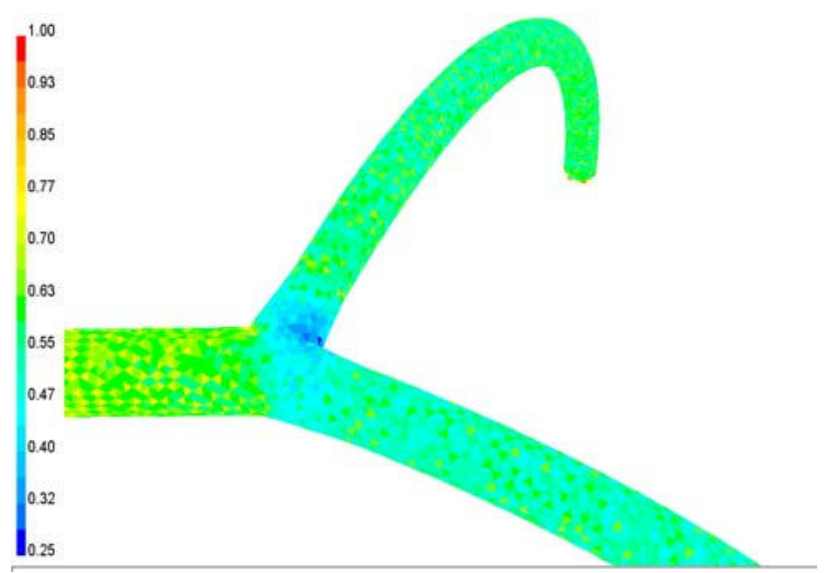

Figure 3. Contours of wall shear stress $\left(\mathrm{N} / \mathrm{m}^{2}\right)$ at point $B$ (15msec)

The same pattern of views is kept for subsequent characteristic points $\mathrm{D}$ and $\mathrm{E}$. In particular, at point $\mathrm{D}$, minimum computed WSS values of the entire bifurcation were of the order $0.51 \mathrm{~N} / \mathrm{m}^{2}$. These values occur at the wall opposite to the branching. Maximum WSS values were of the order $1.26 \mathrm{~N} / \mathrm{m}^{2}$ and occur on the flow divider while the average value in the entire bifurcation region was $0.84 \pm 0.006 \mathrm{~N} / \mathrm{m}^{2}$. Note that point $\mathrm{D}$ exhibits lower average velocity values than point $C$.

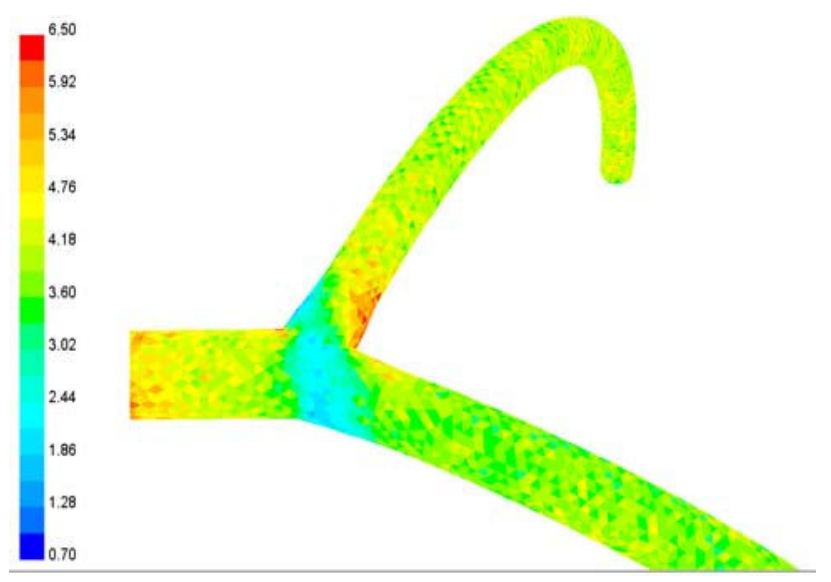

Figure 4. Contours of wall shear stress $\left(\mathrm{N} / \mathrm{m}^{2}\right)$ at point $\mathrm{E}$ (385msec)

Finally, at point E, which occurs $385 \mathrm{msec}$ after point $\mathrm{A}$ at the peak of diastolic left coronary blood flow, the maximum entrance velocity was $0.175 \mathrm{~m} / \mathrm{s}$. Minimum WSS values in this entire bifurcation region were of the order of $0.87 \mathrm{~N} / \mathrm{m}^{2}$. Maximum WSS value was $5.97 \mathrm{~N} / \mathrm{m}^{2}$ while the average value in this region was $3.23 \pm 0.040 \mathrm{~N} / \mathrm{m}^{2}$. These WSS values were the maximum computed throughout the pulsatile waveform.

At point $F(642 \mathrm{msec})$, representing the middle of the cardiological diastole phase, minimum wall shear stress values in the entire bifurcation region were of the order of $0.89 \mathrm{~N} / \mathrm{m}^{2}$. The maximum WSS was $3.74 \mathrm{~N} / \mathrm{m}^{2}$ while the average value in this region was $2.18 \pm 0.023 \mathrm{~N} / \mathrm{m}^{2}$.

\section{Discussion}

Calculated results show that vessel regions located opposite to the flow divider present low wall shear stress (WSS) and low near wall velocity. The blood flow pulsation generates a changing region of flow reversal and separation. Blood flow in arteries is generally considered to be laminar. However, the complex configuration of a vessel promotes flow separation and vortex formation. It is in these complex regions that WSS and wall static pressure greatly fluctuate in magnitude as well as in direction over short distances.

In contrary to earlier studies [3], in the present study the non-Newtonian power law was applied, which is considered to be a more realistic approach. Additional innovative pattern of the current work is considered to be the detailed analysis on particular points of the coronary flow waveform, associated with the physiological and cardiological definitions of pulse cycle. Furthermore, we emphasized in the vulnerable and associated to atherosclerosis regions, by dividing the entire bifurcation region into two distinct regions, namely: the flow divider and the lateral walls. As it is well established [9], the atherosclerosis is localized on the lateral walls, while it spares the flow divider. Thus, taking into account only the entire bifurcation shear stress analyses without spotting the differences between the flow divider and lateral walls could drive us into wrong conclusions. Separating the entire flow region into flow divider and lateral walls and performing a detailed analysis sheds light into localization of atherogenesis. Therefore, such a conception makes the CFD analysis results more attractive and familiar to a physician, rather than to a biomechanical engineer. Finally, the pulse cycle was divided into two hundred time-steps in order to assure complete description of the waveform. Thus, it was possible to depict accurately the high flow gradients of the applied waveform. The description of the waveform is comprised from 3.000 points, ensuring adequate description.

In our results low and oscillatory WSS is found along the lateral walls in accordance to the locations of atheroma in the human left coronary artery. At the inner wall of the left coronary artery, in the region of the flow divider, WSS was highest (systole $=2.69 \mathrm{~N} / \mathrm{m}^{2}$, diastole $=5.97 \mathrm{~N} / \mathrm{m}^{2}$ ) and remained unidirectional during systole, from point $B$ to point $C$. At point of maximum diastole, at the lateral wall of the left coronary artery bifurcation the instantaneous WSS oscillated between 0.87 and $5.82 \mathrm{~N} / \mathrm{m}^{2}$ with mean value $2.83 \pm 0.035 \mathrm{~N} / \mathrm{m}^{2}$. At 
point of maximum systole, also at the lateral wall, the instantaneous WSS oscillated between 0.92 and $2.75 \mathrm{~N} / \mathrm{m}^{2}$ with mean value $1.63 \pm 0.015 \mathrm{~N} / \mathrm{m}^{2}$. From all contours shown in this work, it is evident that there is no significant difference between the patterns of WSS distribution at the bifurcation region at all time steps of the cardiac cycle. Always, the lateral walls exhibit lower WSS than the flow divider. All flows exhibit their minimum values in nearly the same region and show large spatial variations in the WSS magnitude. Note the "ring" and its thickness, which the low WSS distribution forms in the bifurcation region, shown in Figures 3 and 4. All minimum values occur within this "ring". A pulsatile flow intensifies the phenomenon of separation and flow reversal. At the peak of diastole, point E, Figure 4, at the entire bifurcation region, the flow oscillates between 0.87 and $5.97 \mathrm{~N} / \mathrm{m}^{2} \mathrm{WSS}$ values. Thus, it gives a range of values between maximum and minimum of $5.1 \mathrm{~N} / \mathrm{m}^{2}$. This is the highest range of oscillations for all tested time steps. The average WSS value is $3.23 \pm 0.040 \mathrm{~N} / \mathrm{m}^{2}$. During the cardiac cycle, flow stagnation and flow reversal throughout the epicardial left coronary artery appears only at the onset of the physiological systole, from point A to point B. In this time period WSS and velocity attain the lowest values of the complete cycle. On the whole, coronary WSS in systole is significantly lower than in diastole. Given the well-established importance of low shear stress in atherogenesis, it seems reasonable to associate the systolic period with the development of atherosclerotic plaques rather than the diastolic one. On the other hand, in resting conditions, diastole lasts more than systole, henceforth compensating the balance towards higher wall shear values.

Moreover, the steep WSS gradient appearing after incisura occurrence, point $\mathrm{D}$, further reduces the residence time and the contact of blood particles with the endothelium, thus contributing to the wash out of atherogenous materials.

\section{Conclusion}

The distribution of low WSS along the walls is in agreement with the common locations of atheroma. It is the systolic period, rather than the diastolic one, which is probably, associated with the development of atherosclerotic plaques, due to significantly lower WSS values, at the lateral walls of the bifurcation, during systole. On the contrary, significantly higher WSS values occur during diastole. Diastole, lasting more than systole, compensates the balance towards higher WSS values, further reducing the contact time of blood particles with the endothelium, thus exerting beneficial role. Bradycardia, prolonging diastole against systole, act as a benefector, inhibiting coronary atherogenesis.

\section{References}

[1] Tu C, Deville M, Dheur L, Vanderschuren L. Finite element simulation of pulsatile flow through arterial stenosis. J Biomech. 1992;25(10):1141-52.

[2] $\mathrm{He} \mathrm{X}, \mathrm{Ku} \mathrm{DN}$. Pulsatile flow in the human left coronary artery bifurcation: average conditions. J Biomech Eng 1996;118(1):74-82.

[3] Gijsen, FJ, Allanic E, van de Vosse FN, Janssen JD. The influence of the non-Newtonian properties of blood on the flow in large arteries: unsteady flow in a 90 degrees curved tube. J Biomech 1999;32(7):705-13.

[4] Giannoglou GD, Soulis JV, Farmakis TM, Louridas GE. Near wall viscosity distribution between inner and outer right coronary artery wall. In: Lewis BS, Halon DA, Flugelman MY, Touboul P, editors. Coronary Artery Disease. Prevention to Intervention. Proceedings of the $3^{\text {rd }}$ International Congress on Coronary Artery Disease. Bologna: Monduzzi Editore-International Proceedings DIVISION, 2000:221-26.

[5] Tang TD. Periodic flow in a bifurcation tube at moderate Reynolds number. Thesis (PhD). Atlanta: Georgia Institute of Technology, 1990.

[6] Nerem RM, Seed WA. Coronary artery geometry and its fluid mechanical implications. In: Schettler $\mathrm{G}$ et al, editors. Fluid Dynamics as a Localizing Factor for Atherosclerosis. Berlin: Springer-Verlag, 1983.

[7] Macalpin RN, Abbasi AS, Grollman JH Jr, Eber L. Human coronary artery size during life. A cinearteriographic study. Radiology 1973;108(3): 567-76.

[8] Grottum P, Svindland A, Walloe L. Localization of atherosclerotic lesions in the bifurcation of the main left coronary artery. Atherosclerosis 1983;47(1):55-62.

[9] Kjaernes M, Svindland A, Walloe L, Wille SO. Localization of early atherosclerotic lesions in an arterial bifurcation in humans. Acta Pathol Microbiol Scand [A] 1981;89(1):35-40.

Address for correspondence.

George D Giannoglou

Ath. Soulioti 17 Str., Thessaloniki 54642

yan@med.auth.gr 